PROCEEDINGS OF THE

AMERICAN MATHEMATICAL SOCIETY

Volume 130, Number 6, Pages 1843-1850

S 0002-9939(02)06512-7

Article electronically published on January 25, 2002

\title{
LINEARITY OF DIMENSION FUNCTIONS FOR SEMILINEAR $G$-SPHERES
}

\author{
IKUMITSU NAGASAKI
}

(Communicated by Ralph Cohen)

Dedicated to the memory of Professor Katsuo Kawakubo

\begin{abstract}
In this paper, we show that the dimension function of every semilinear $G$-sphere is equal to that of a linear $G$-sphere for finite nilpotent groups $G$ of order $p^{n} q^{m}$, where $p, q$ are primes. We also show that there exists a semilinear $G$-sphere whose dimension function is not virtually linear for an arbitrary nonsolvable compact Lie group $G$.
\end{abstract}

\section{InTRODUCTION}

Let $G$ be a compact Lie group and $V$ an orthogonal $G$-representation. The unit sphere $S(V)$ of $V$ is a closed $G$-manifold whose $H$-fixed point set is a sphere (or possibly the empty set) for every closed subgroup $H$ of $G$. We call this $G$-manifold a linear $G$-sphere. A semilinear $G$-sphere is defined as a natural generalization of a linear $G$-sphere, namely it is a (smooth) closed $G$-manifold such that the $H$-fixed point set is a homotopy sphere or the empty set for every closed subgroup $H$ of $G$.

Many authors studied linear $G$-spheres, in particular K. Kawakubo [9] studied the $G$-homotopy classification of linear $G$-spheres for compact abelian groups, and T. tom Dieck [3] studied these spheres for finite $p$-groups.

T. tom Dieck and T. Petrie [6] introduced the notion of $G$-homotopy representations and developed the (stable) homotopy theory of $G$-homotopy representations. E. Laitinen [10] developed the unstable theory of $G$-homotopy representations. The $G$-homotopy representation $X$ is defined as a $G$-CW complex satisfying the following properties (1)-(3):

(1) For every closed subgroup $H$, the $H$-fixed point set $X^{H}$ is homotopy equivalent to a sphere $S^{\underline{n}}(H)-1$, where we define the (-1)-dimensional sphere $S^{-1}$ as the empty set.

(2) $\underline{n}(H)-1=\operatorname{dim} X^{H}$ for every closed subgroup $H$. (We set $\operatorname{dim} \emptyset=-1$.)

(3) $X$ has finitely many orbit types.

Note that a semilinear $G$-sphere is an example of a $G$-homotopy representation since any (smooth) $G$-manifold has a $G$-CW complex structure. We say that a

Received by the editors March 20, 2000.

2000 Mathematics Subject Classification. Primary 57S25; Secondary 57S15, 57S17.

Key words and phrases. Dimension function, semilinear $G$-sphere, homotopy representation.

This work was partially supported by Grant-in-Aid for Scientific Research. 
$G$-homotopy representation $X$ is linear if $X$ is $G$-homotopy equivalent to a linear $G$-sphere.

There exist many nonlinear $G$-homotopy representations for groups $G$. (See for example [16], [17].)

For any $G$-homotopy representation $X$, we define the integer-valued function $\operatorname{Dim} X$ on the set of closed subgroups by setting $\operatorname{Dim} X(H)=\operatorname{dim} X^{H}+1$ for $H \leq G$. This function is called the dimension function of $X$. Clearly the dimension function is a $G$-homotopy invariant, and the additivity of dimension functions holds, i.e., $\operatorname{Dim} X * Y=\operatorname{Dim} X+\operatorname{Dim} Y$ for $G$-homotopy representations, where $*$ means join. (Note that $X * Y$ is also a $G$-homotopy representation up to $G$-homotopy.) Dimension functions of $G$-homotopy representations are studied by [4 and [2] for finite groups and by [1] for compact Lie groups. We say that the dimension function $\underline{n}$ is linear if $\underline{n}=\operatorname{Dim} S(V)$ for some $G$-representation $V$, and that $\underline{n}$ is virtually linear (or stably linear) if $\underline{n}=\operatorname{Dim} S(V)-\operatorname{Dim} S(W)$ for some $V$ and $W$.

The purpose of this paper is to study the dimension functions of semilinear $G$-spheres from the viewpoint of linearity.

For certain groups $G$, it is known that a semilinear $G$-sphere with nonlinear dimension function is constructed by the equivariant surgery. (See Remark 1.3 below.) On the other hand, the following result is known for $p$-groups.

Theorem 1.1 (7], 4]). For any p-group P, every P-homotopy representation has a linear dimension function.

Our first result is the following.

Theorem A. Let $G$ be a finite nilpotent group of order $p^{n} q^{m}$, where $p$, $q$ are primes. Then every semilinear $G$-sphere has a linear dimension function.

Remark 1.2. Theorem A does not hold for $G$-homotopy representations unless $G$ is a $p$-group.

Remark 1.3. Further generalization of Theorem A cannot be expected, because the following results are known.

(1) There exists a semilinear $C_{p q r}$-sphere with nonlinear dimension function for cyclic groups $C_{p q r}$ of order $p q r$, where $p, q, r$ are distinct odd primes (14], [11).

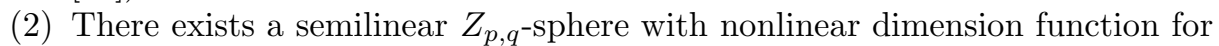
metacyclic groups $Z_{p, q}$ of order $p q$, where $p, q$ are distinct odd primes and $q \mid(p-1)([13)$.

(3) There exists a semilinear $S^{1}$-sphere with nonlinear dimension function for the circle group $S^{1}$ ([15]).

We also show the following result on nonlinearity of dimension functions in Section 4.

Theorem B. Let $G$ be an arbitrary nonsolvable compact Lie group. Then there exists a semilinear $G$-sphere whose dimension function is not virtually linear.

\section{The CASE of $C_{p q}$}

We first note the following fact.

Lemma 2.1. Let $G$ be a compact Lie group. If a semilinear $G$-sphere $\Sigma$ has a $G$-fixed point, then $\Sigma$ is linear, i.e., G-homotopy equivalent to a linear $G$-sphere, and in particular its dimension function is linear. 
Proof. Let $x$ be a $G$-fixed point of $\Sigma$. Take a (small) $G$-invariant neighborhood $U$ of $x$ which is $G$-diffeomorphic to Int $D(V)$, where $V$ is the tangential representation at $x$. Then the projection $\Sigma \rightarrow \Sigma /(\Sigma-U) \cong S(V \oplus \mathbb{R})$ is a $G$-homotopy equivalence by the equivariant Whitehead theorem.

In this section, we show the following special case of Theorem A.

Proposition 2.2. Let $G$ be a cyclic group $C_{p q}$ of order $p q$, where $p, q$ are primes. Then every semilinear $G$-sphere has a linear dimension function.

Proof. In case $p=q$, by Theorem 1.1, every semilinear $G$-sphere has a linear dimension function. We may assume $p>q$. By Lemma 2.1, we may also assume that the action is $G$-fixed point free. Let $\Sigma$ be a semilinear $C_{p q}$-sphere without a $C_{p q}$-fixed point, and let $\underline{n}$ be the dimension function of $\Sigma$.

We first show the inequality $\underline{n}\left(C_{p}\right)+\underline{n}\left(C_{q}\right) \leq \underline{n}(1)$. Put $M:=\Sigma^{C_{q}}$, which is a homotopy sphere with free $C_{p}\left(\cong C_{p q} / C_{q}\right)$-action. Put $N:=\Sigma-N\left(\Sigma^{C_{p}}\right)$, where $N\left(\Sigma^{C_{p}}\right)$ is an equivariant open tubular neighborhood of $\Sigma^{C_{p}}$. Then $N$ is considered as a (compact) $C_{p}$-manifold with free $C_{p}$-action and its homology group $H_{*}(N ; \mathbb{Z})$ is isomorphic to $H_{*}\left(S^{\underline{n}}(1)-\underline{n}\left(C_{q}\right)-1 ; \mathbb{Z}\right)$ by the Alexander duality. Clearly the natural inclusion $i: M \rightarrow N$ is a $C_{p}$-map. If necessary, by taking the join of $S(W)$ and $N, N * S(W)$ is simply connected and $\operatorname{dim} N * S(W) \geq 3$, where $W$ is a free $C_{p^{-}}$ representation of dimension $m>2$. Then $N * S(W)$ is a $C_{p}$-CW complex which is (nonequivariantly) homotopy equivalent to $S^{\underline{n}}\left(C_{q}\right)+m-1$. By Proposition 5.9 of [6], one can see that $N * S(W)$ is $C_{p}$-homotopy equivalent to a free $C_{p}$-homotopy representation $X$ of dimension $\underline{n}(1)-\underline{n}\left(C_{q}\right)+m-1$, and $M * S(W)$ is also a free $C_{p}$-homotopy representation of dimension $\underline{n}\left(C_{p}\right)+m-1$. Let $f: N * S(W) \rightarrow X$ be a $C_{p}$-homotopy equivalence. Then the composite $f \circ(i * i d): M * S(W) \rightarrow$ $N * S(W) \rightarrow X$ is a $C_{p}$-map between free $C_{p}$-homotopy representations. By the Borsuk-Ulam theorem for free $C_{p}$-actions (cf. [10, Proposition 1.14]), we obtain $\underline{n}\left(C_{p}\right)+m-1 \leq \underline{n}(1)-\underline{n}\left(C_{q}\right)+m-1$, and hence $\underline{n}\left(C_{p}\right)+\underline{n}\left(C_{q}\right) \leq \underline{n}(1)$.

Next, since $C_{p q / r}=\bar{C}_{p q} / C_{r}(r=p$ or $q)$ acts freely on the homotopy sphere $\Sigma^{C_{r}}, \underline{n}\left(C_{r}\right)=\operatorname{dim} \Sigma^{C_{r}}+1$ is even unless $p q / r=2$. Then there exists a free $C_{p q / r^{-}}$ representation $V_{r}$ such that $\operatorname{dim} V_{r}=\underline{n}\left(C_{r}\right)$. Via the projection $C_{p q} \rightarrow C_{p q / r}$, we regard $V_{r}$ as a $C_{p q}$-representation with kernel $C_{r}$. Put $k:=\underline{n}(1)-\underline{n}\left(C_{p}\right)-\underline{n}\left(C_{q}\right) \geq 0$. Then $k$ is even. In fact, since $C_{p}\left(\leq C_{p q}\right)$ ( $p$ :odd prime by the assumption $p>q$ ) acts semi-freely on $\Sigma$, it follows that $\underline{n}(1)-\underline{n}\left(C_{p}\right)$ is even, and $\underline{n}\left(C_{q}\right)$ is even as mentioned above. Therefore $k$ is even and so we can take a free $C_{p q}$-representation $U$ of dimension $k$. Put $V=V_{p} \oplus V_{q} \oplus U$. One can easily see that $\operatorname{Dim} \Sigma=\operatorname{Dim} S(V)$.

\section{The General CASE}

Throughout this section, $G$ is a finite group. We first recall well-known results on linear dimension functions. Let $F(G)$ be the free module consisting of integervalued functions on the set of subgroups of $G$. Let us denote by $D_{+}^{\ell}(G)$ the set of linear dimension functions for $G$, and by $D^{\ell}(G)$ the submodule of $F(G)$ generated by $D_{+}^{\ell}(G)$. By the additivity of dimension functions, any function in $D^{\ell}(G)$ has a form of $\underline{n}-\underline{n}^{\prime}$, where $\underline{n}, \underline{n}^{\prime}$ are linear dimension functions, and hence $D^{\ell}(G)$ is the free module consisting of virtually linear dimension functions for $G$.

We say that $G$-representations $V$ and $W$ are $G$-Galois conjugate if there exists an integer $k$ prime to $|G|$ such that $\chi_{V}(g)=\chi_{W}\left(g^{k}\right)$ for all $g \in G$, where $\chi_{V}$ denotes the character of $V$. Note that if $V$ and $W$ are $G$-Galois conjugate, then 
$\operatorname{Dim} S(V)=\operatorname{Dim} S(W)$ and $\operatorname{Ker} V=\operatorname{Ker} W$. Let $V_{1}, V_{2}, \ldots, V_{r}$ be the complete representative system of $G$-Galois conjugate classes of irreducible $G$-representations, and $\mathcal{B}(G)$ the set of dimension functions of $S\left(V_{i}\right)$.

Proposition $3.1\left([12,[5])\right.$. The set $\mathcal{B}(G)$ is a $\mathbb{Z}$-basis of $D^{\ell}(G)$.

We call $\mathcal{B}(G)$ the standard basis of $D^{\ell}(G)$. By Proposition 3.1, any virtually linear function $\underline{n}$ is presented as

$$
\underline{n}=\sum_{\underline{m} \in \mathcal{B}(G)} a(\underline{m}, \underline{n}) \underline{m} .
$$

We call the integer $a(\underline{m}, \underline{n})$ the multiplicity of $\underline{m}$ in $\underline{n}$. We also put

$$
\mathcal{B}(G, \underline{n})=\{\underline{m} \in \mathcal{B}(G) \mid a(\underline{m}, \underline{n}) \neq 0\} .
$$

We define $\operatorname{Ker} \underline{n}$ as $\operatorname{Ker} V$ if $\underline{n}=\operatorname{Dim} S(V)$. For any $\underline{n} \in D^{\ell}(G)$, we define a function $\underline{n}^{K}$ by setting $\underline{n}^{K}(H / \bar{K})=\underline{n}(H)$ for $H / K \leq G / \bar{K}$, where $K$ is any normal subgroup of $G$. Then $\underline{n}^{K} \in D^{\ell}(G / \bar{K})$ because $\underline{n}^{K}=\operatorname{Dim} S\left(V^{K}\right)-\operatorname{Dim} S\left(W^{K}\right)$ if $\underline{n}=\operatorname{Dim} S(V)-\operatorname{Dim} S(W)$. We shall show the following lemmas.

Lemma 3.2. Let $K$ be an arbitrary normal subgroup of $G$. If $\underline{m} \in \mathcal{B}(G)$ and Ker $\underline{m} \geq K$, then $\underline{m}^{K}=0$ (zero-function).

Proof. Let $\underline{m}=\operatorname{Dim} S(V)$, where $V$ is an irreducible $G$-representation. Then $V^{K}$ is regarded as a $G$-subrepresentation of $V$, and $V^{K} \neq V$ by $\operatorname{Ker} \underline{m} \geq K$. Since $V$ is irreducible, we have $V^{K}=0$, and hence $\underline{m}^{K}=0$.

Put $B=\left\{\underline{m}^{K} \mid \underline{m} \in \mathcal{B}(G)\right.$ such that $\left.\operatorname{Ker} \underline{m} \geq K\right\}$.

Lemma 3.3. The set $B$ is a part of the standard basis $\mathcal{B}(G / K)$.

Proof. If $V$ is an irreducible $G$-representation whose kernel contains $K$, then $V^{K}$ (= $V)$ is an irreducible $G / K$-representation. It is easy to see that irreducible $G$ representations $V$ and $W$ are $G$-Galois conjugate if and only if $V^{K}$ and $W^{K}$ are $G / K$-Galois conjugate. This shows the desired result.

We now prove Theorem A. Since $G$ is nilpotent of order $p^{n} q^{m}$, we may put $G=G_{p} \times G_{q}$, where $G_{p}$ [resp. $\left.G_{q}\right]$ is a $p$ - [resp. $q$-] group. Let $\underline{n}$ be the dimension function of a semilinear $G$-sphere. Since all dimension functions of $G$-homotopy representations are virtually linear if (and only if) $G$ is nilpotent ([6], Proposition $10.23), \underline{n}$ is presented as a linear combination of $\underline{m} \in \mathcal{B}(G) ; \underline{n}=\sum_{\underline{m} \in \mathcal{B}(G)} a(\underline{m}, \underline{n}) \underline{m}$. In order to prove Theorem A, it suffices to show that all $a(\underline{m}, \underline{n})$ are nonnegative.

The proof is done by induction on the order of $G$. For any $\underline{m} \in \mathcal{B}(G, \underline{n})$ with nontrivial kernel $K$, it is seen that $a(\underline{m}, \underline{n})=a\left(\underline{m}^{K}, \underline{n}^{K}\right)$ by Lemmas 3.2 and 3.3 . and hence $a(\underline{m}, \underline{n})$ is nonnegative by the inductive assumption.

We have to show that $a(\underline{m}, \underline{n})$ is nonnegative when $\operatorname{Ker} \underline{m}=1$. By Theorem 1.1 Theorem A holds for $p$-groups, and so we assume that $p$ and $q$ are distinct primes and that $G_{p}$ and $G_{q}$ are nontrivial.

The proof is divided into two cases: (1) $G$ has a normal subgroup $A$ isomorphic to $C_{p} \times C_{p}$ or $C_{q} \times C_{q}$, and (2) otherwise.

Case (1): If there is no $\underline{m} \in \mathcal{B}(G, \underline{n})$ with trivial kernel, there is nothing to do. We assume that there is an $\underline{m} \in \mathcal{B}(G, \underline{n})$ with trivial kernel, namely $G$ has a faithful irreducible $G$-representation. Let $\underline{m}=\operatorname{Dim} S(U)$, where $U$ is a faithful irreducible $G$-representation. By assumption, $G$ has a normal subgroup $A$ isomorphic to $C_{p} \times$ 
$C_{p}$ or $C_{q} \times C_{q}$. We may assume that $A$ is isomorphic to $C_{p} \times C_{p}$. Let $A_{0}, A_{1}$, $\ldots, A_{p}$ be all subgroups of order $p$ in $A$. By representation theory, the following is known.

Proposition 3.4. Under the above situation,

(1) One of the $A_{i}$, say $A_{0}$, is normal in $G$ and the others have the same normalizer $N=N_{G}\left(A_{i}\right)$ of index $p$ in $G$. Furthermore $A_{0}$ is in the center $Z(G)$ of $G$, and $N$ is normal in $G$ and contains $A$.

(2) The restriction $\operatorname{Res}_{N} U$ is the direct sum of irreducible $N$-representations $U_{1}, \ldots, U_{p}$ such that $\operatorname{Ker}_{\operatorname{Res}_{A}} V_{i}=A_{i}(1 \leq i \leq p)$.

(3) The irreducible summands $U_{i}$ and $U_{j}$ are not $N$-Galois conjugate for $i \neq j$.

(4) Let $W$ be another faithful irreducible $G$-representation and let $\operatorname{Res}_{N} W=$ $W_{1} \oplus \cdots \oplus W_{p}$ as in (2). If $U$ and $W$ are not $G$-Galois conjugate, then $U_{i}$ and $W_{j}$ are not $N$-Galois conjugate for every $i$ and $j$.

Proof. Statements (1) and (2) are shown by Proposition 5.12 of [5] and its proof. Statements (3) and (4) are shown by Proposition 5.6 of [18].

For any $\underline{n} \in D^{\ell}(G)$ and any subgroup $H$ of $G$, we define the restriction $r_{H} \underline{n} \in$ $D^{\ell}(H)$ by setting $r_{H} \underline{n}(L)=\underline{n}(L)$ for $L \leq H$. Put $\underline{m}_{i}=\operatorname{Dim} S\left(U_{i}\right) \in \mathcal{B}(N)$ $(1 \leq i \leq p)$. By Proposition $3.4 r_{N} \underline{m}=\sum_{i} \underline{m}_{i}$.

Lemma 3.5. Under the above situation, $a(\underline{m}, \underline{n})=a\left(\underline{m}_{i}, r_{N} \underline{n}\right)(1 \leq i \leq p)$.

Proof. Since $\underline{n}=\sum_{\underline{\ell \in B}(G, \underline{n})} a(\underline{\ell}, \underline{n}) \underline{\ell}$, it follows from the additivity of the restriction that

$$
a\left(\underline{m}_{i}, r_{N} \underline{n}\right)=\sum_{\underline{\ell \in \mathcal{B}(G, \underline{n})}} a(\underline{\ell}, \underline{n}) a\left(\underline{m}_{i}, r_{N} \underline{\ell}\right) .
$$

We first consider the case where $\underline{\ell} \in \mathcal{B}(G, \underline{n})$ has a nontrivial kernel $K$. We note that the center $Z(G)=Z\left(G_{p}\right) \times Z\left(G_{q}\right)$ of $G$ is cyclic since $G$ has a faithful irreducible $G$-representation (see for example [8]). Furthermore any (nontrivial) $p$ group $P$ has a nontrivial center, and any nontrivial normal subgroup of $P$ intersects the center $Z(P)$ nontrivially. Since $N=N_{G_{p}}\left(A_{i}\right) \times G_{q}$, Ker $r_{N} \underline{\ell}=N \cap K$ is a nontrivial normal subgroup of $G$, and hence $\operatorname{Ker} \underline{s} \geq N \cap K$ for any $\underline{s} \in \mathcal{B}\left(N, r_{N} \underline{\ell}\right)$. On the other hand

$$
r_{N / N \cap K}\left(\underline{m}^{N \cap K}\right)=\left(r_{N} \underline{m}\right)^{N \cap K}=\sum_{i} \underline{m}_{i}^{N \cap K} .
$$

Since $\underline{m}$ has the trivial kernel, it follows from Lemma 3.2 that $m^{N \cap K}=0$, and hence $\underline{m}_{i}^{N \cap K}=0$ for $1 \leq i \leq p$. Thus we have Ker $\underline{m}_{i} ¥ N \cap K$ and Ker $\underline{s} \neq \operatorname{Ker} \underline{m}_{i}$. This shows that $a\left(\underline{m}_{i}, r_{N} \underline{\ell}\right)=0$ when $\operatorname{Ker} \underline{\ell} \neq 1$.

Next, if $\underline{\ell} \in \mathcal{B}(G, \underline{n})$ has the trivial kernel, by Proposition 3.4 (3) and (4),

$$
a\left(\underline{m}_{i}, r_{N} \underline{\ell}\right)= \begin{cases}1 & \text { if } \underline{\ell}=\underline{m} \\ 0 & \text { if } \underline{\ell} \neq \underline{m} .\end{cases}
$$

Thus we obtain $a(\underline{m}, \underline{n})=a\left(\underline{m}_{i}, r_{N} \underline{n}\right)(1 \leq i \leq p)$ from equation (3.1).

By Lemma 3.5 and the induction hypothesis, we see that $a(\underline{m}, \underline{n})$ is nonnegative for any $\underline{m}$ with trivial kernel. Thus the inductive argument in Case (1) is completed.

Case (2): Assume $p<q$. In this case, as is well known (see [8]), $G_{p}$ is cyclic, dihedral, quaternion or semi-dihedral, where the last three cases occur only in 
case $p=2$, and $G_{q}$ is cyclic. All faithful irreducible $G_{r}$-representations $(r=p, q)$ are $G_{r}$-Galois conjugate by investigating the character tables, and hence all faithful irreducible $G$-representations are $G$-Galois conjugate. Therefore $\mathcal{B}(G)$ contains only one dimension function with trivial kernel. We denote by $\underline{u}$ this dimension function. Let $C$ be a cyclic subgroup $C=C_{p} \times C_{q}$ of order $p q$ in the center $Z(G)$. Then $\mathcal{B}(C)$ consists of four elements $\underline{\ell}_{1}, \underline{\ell}_{2}, \underline{\ell}_{3}, \underline{\ell}_{4}$ whose kernels are $1, C_{p}, C_{q}, C$ respectively. We have to show that $a(\underline{u}, \underline{n})$ is nonnegative.

Lemma 3.6. Under the above situation, $a\left(\underline{\ell}_{1}, r_{C} \underline{n}\right)=a(\underline{u}, \underline{n}) a\left(\underline{\ell}_{1}, r_{C} \underline{u}\right)$.

Proof. The dimension function $\underline{n}$ is presented as $\underline{n}=\sum_{\underline{m} \in \mathcal{B}(G, \underline{n})} a(\underline{m}, \underline{n}) \underline{m}$, and furthermore $r_{C} \underline{n}=\sum_{\underline{m} \in \mathcal{B}(G, \underline{n})} a(\underline{m}, \underline{n}) r_{C} \underline{m}$ and $r_{C} \underline{m}=\sum_{i} a\left(\underline{\ell}_{i}, r_{C} \underline{m}\right) \underline{\ell}_{i}$. If $\underline{m} \in$ $\mathcal{B}(G, \underline{n})$ has a nontrivial kernel $K$, then $\operatorname{Ker} r_{C} \underline{m}=C \cap K$ is nontrivial since $K$ is a nontrivial normal subgroup of $G$ and $C=C_{p} \times C_{q}$ is contained in the center $Z(G)$. (Note that $Z\left(G_{r}\right)(r=p, q)$ is a cyclic $r$-group.) Hence we have $a\left(\underline{\ell}_{1}, r_{C} \underline{m}\right)=0$ by Lemma 3.2. and so

$$
\begin{aligned}
a\left(\underline{\ell}_{1}, r_{C} \underline{n}\right) & =\sum_{\underline{m} \in \mathcal{B}(G, \underline{n})} a(\underline{m}, \underline{n}) a\left(\underline{\ell}_{1}, r_{C} \underline{m}\right) \\
& =a(\underline{u}, \underline{n}) a\left(\underline{\ell}_{1}, r_{C} \underline{u}\right) .
\end{aligned}
$$

Lemma 3.7. The number $a\left(\underline{\ell}_{1}, r_{C} \underline{u}\right)$ is positive.

Proof. Suppose $r_{C} \underline{u}=\sum_{i=1}^{4} b_{i} \underline{\ell}_{i}$, where $b_{i}=a\left(\underline{\ell}_{i}, r_{C} \underline{u}\right)$. Since Ker $\underline{u}=1,\left(r_{C} \underline{u}\right)^{C}=$ $r_{C / C} \underline{u}^{C}=0$ and $\left(r_{C} \underline{u}\right)^{C_{r}}=r_{C / C_{r}} \underline{u}^{C_{r}}=0$ for $r=p$ and $q$. This shows $b_{2}=b_{3}=$ $b_{4}=0$, and hence $r_{C} \underline{u}=b_{1} \underline{\ell}_{1}$. Since $r_{C} \underline{u}(1)$ and $\underline{\ell}_{1}(1)$ are positive, it follows that $b_{1}=a\left(\underline{\ell}_{1}, r_{C} \underline{u}\right)$ is positive.

By Lemmas 3.6 and 3.7 and the inductive assumption, we obtain that $a(\underline{u}, \underline{n})$ is nonnegative. Thus the inductive argument in Case (2) is completed and Theorem A has been proved.

\section{Semilinear $G$-SPheres With NOnlinear Dimension FUnCtions}

In this section we prove Theorem B. Let $G$ be a compact Lie group. For the sake of the proof, we shall show the following.

Lemma 4.1. Let $\underline{n}$ be a virtually linear dimension function. If $\underline{n}(C)=0$ for every (finite) cyclic subgroup $C$ of $G$, then $\underline{n}$ is a zero-function.

Proof. Let $\underline{n}=\operatorname{Dim} S\left(V_{1}\right)-\operatorname{Dim} S\left(V_{2}\right)$. In the case where $G$ is finite, by representation theory, $\underline{n}(H)=\operatorname{dim} V_{1}^{H}-\operatorname{dim} V_{2}^{H}=\sum_{g \in H}\left(\chi_{V_{1}}(g)-\chi_{V_{2}}(g)\right)$, where $\chi_{V_{i}}$ is the character of $V_{i}$. We also note

$$
\sum_{g \in H}\left(\chi_{V_{1}}(g)-\chi_{V_{2}}(g)\right)=\sum_{\substack{C \leq H \\ C: \text { cyclic }}} \sum_{g \in C^{*}}\left(\chi_{V_{1}}(g)-\chi_{V_{2}}(g)\right),
$$

where $C^{*}$ denotes the set of generators of $C$. We set, for any cyclic subgroup $C$,

$$
\begin{aligned}
& h(C)=\sum_{g \in C}\left(\chi_{V_{1}}(g)-\chi_{V_{2}}(g)\right), \\
& k(C)=\sum_{g \in C^{*}}\left(\chi_{V_{1}}(g)-\chi_{V_{2}}(g)\right) .
\end{aligned}
$$


From equation (4.1), we obtain

$$
h(C)=\sum_{D \leq C} k(D) .
$$

Applying the Möbius inversion to 4.2, we obtain

$$
k(C)=\sum_{D \leq C} \mu(|C / D|) h(D),
$$

where $\mu$ is the Möbius function. Since, by assumption, $h(D)=\underline{n}(D)=0$ for any cyclic subgroup $D$, we obtain $k(C)=0$ for any cyclic subgroup $C$. It follows from (4.1) that $\underline{n}(H)=0$.

We next consider the case where $G$ is a compact Lie group. Since $G_{0}$ is normal, $V_{i}^{G_{0}}$ is considered as a $G$-subrepresentation of $V_{i}$. We denote by $V_{i} G_{0}$ the orthogonal complement of $V_{i}^{G_{0}}$ in $V_{i}$. Since $V_{1} G_{0}$ and $V_{2} G_{0}$ are isomorphic as $G$-representations by Theorem 1.1 of [20], we have $\underline{n}=\operatorname{Dim} S\left(V_{1}^{G_{0}}\right)-\operatorname{Dim} S\left(V_{2}^{G_{0}}\right)$, and also $\underline{n}^{G_{0}}=$ $\operatorname{Dim} S\left(V_{1}^{G_{0}}\right)-\operatorname{Dim} S\left(V_{2}^{G_{0}}\right)$ by considering $V_{i}^{G_{0}}$ as a $G / G_{0}$-representation. Let $K / G_{0}$ be any cyclic subgroup of $G / G_{0}, g \in K$ an element representing a generator of $K / G_{0}$, and $T$ the closure of the subgroup generated by $g$. Then $K / G_{0}=$ $T G_{0} / G_{0}$, and $\underline{n}^{G_{0}}\left(K / G_{0}\right)=\underline{n}(T)$. Since $\bigcup_{C \leq T, C \text { :finite cyclic }} C$ is dense in $T$ and a $G$-representation has only finitely many isotropy types, there exists a finite cyclic subgroup $C$ such that $\underline{n}(T)=\underline{n}(C)$. By assumption, $\underline{n}(C)=0$ and hence $\underline{n}^{G_{0}}\left(K / G_{0}\right)=0$. Since this lemma has been proved for finite groups, by applying to $\underline{n}^{G_{0}}$, we obtain $\underline{n}(H)=\underline{n}^{G_{0}}\left(H G_{0} / G_{0}\right)=0$ for every closed subgroup $H$.

By [19, there exists a smooth $G$-action on a disk $D$ such that $D^{H}$ is a disk if $H$ is solvable, and the empty set if $H$ is nonsolvable. In particular $D$ has no $G$-fixed point if $G$ is nonsolvable. We call $D$ a quasilinear $G$-disk. Clearly $D \times D^{n}$ is also a quasilinear $G$-disk, where $D^{n}$ is an $n$-dimensional disk with trivial $G$-action, and the boundary $\partial\left(D \times D^{n}\right)$ is a semilinear $G$-sphere. We put $\Sigma_{n}=\partial\left(D \times D^{n}\right)$.

The following leads to the proof of Theorem B.

Theorem 4.2. Let $G$ be a nonsolvable compact Lie group. Then the dimension function of $\Sigma_{n}$ is not virtually linear except at most one $n$.

Proof. Suppose that $\operatorname{Dim} \Sigma_{r}$ and $\operatorname{Dim} \Sigma_{s}$ are virtually linear for two distinct numbers $r, s(r<s)$. Put $\underline{n}=\operatorname{Dim} \Sigma_{r}, \underline{m}=\operatorname{Dim} \Sigma_{s}$ and $\underline{\ell}=\operatorname{Dim} S\left(\mathbb{R}^{s-r}\right)$. Then $\underline{u}:=\underline{m}-\underline{n}-\underline{\ell}$ is also a virtually linear dimension function. For any finite cyclic subgroup $\bar{C}, \underline{n}(C)=\operatorname{Dim} \partial D(C)+r, \underline{m}(C)=\operatorname{Dim} \partial D(C)+s$ and $\underline{\ell}(C)=s-r$. Therefore $\underline{u}(C)=0$. By Lemma 4.1, we have $\underline{u}=0$. On the other hand, since $\Sigma_{\ell}^{G}$ $(\ell=r, s)$ is empty, we have $\underline{u}(G)=s-r \neq 0$. This is a contradiction.

\section{REFERENCES}

[1] S. Bauer, Dimension functions of homotopy representations for compact Lie groups, Math. Ann. 280 (1988), 247-265. MR 89e:57030

[2] — A linearity theorem for group actions on spheres with applications to homotopy representations, Comment. Math. Helv. 64 (1989), 167-172. MR 90e:57067

[3] T. tom Dieck, Homotopy equivalent group representations, J. Reine Angew. Math. 298 (1978), 182-195. MR 58:18498

[4] , Homotopiedarstellungen endlich Gruppen: Dimensionsfunktionen, Invent. Math. 67 (1982), 231-252. MR 84b:57029

[5] — Transformation groups, Walter de Gruyter, Berlin, New York, 1987. MR 89c:57048 
[6] T. tom Dieck and T. Petrie, Homotopy representations of finite groups, Inst. Hautes Etudes Sci. Publ. Math. 56 (1982), 129-169. MR 86b:57020

[7] R. M. Dotzel and G. C. Hamrick, p-group actions on homology spheres, Invent. Math. 62 (1981), 437-442. MR 82j:57037

[8] D. Gorenstein, Finite groups, Harper \& Row, New York, Evanston and London, 1968. MR 38:229

[9] K. Kawakubo, Equivariant homotopy equivalence of group representations, J. Math. Soc. Japan 32 (1980), 105-118. MR 82a:57034

[10] E. Laitinen, Unstable homotopy theory of homotopy representations, Lecture Notes in Math. 1217 (1985), 210-248. MR 88i:57013

[11] E. Laitinen and M. Raussen, Homotopy types of locally linear representation forms, Manuscripta Math. 88 (1995), 33-52. MR 97g:57056

[12] C. N. Lee and A. G. Wasserman, On the group $J O(G)$, Mem. Amer. Math. Soc. 159 (1975). MR 51:6808

[13] C. Mackrodt, Representation forms for metacyclic groups, Manuscripta Math. 73 (1991), 261-287. MR 93c:57032

[14] I. Madsen and M. Raussen, Locally linear representation forms, Osaka J. Math. 27 (1990), 567-591. MR 92k:57067

[15] D. Montgomery and C. T. Yang, Differentiable pseudo-free circle actions on homotopy seven spheres, Second conference of compact transformation groups, Lecture Notes in Math. 298 (1972), 41-101. MR 50:14825

[16] I. Nagasaki, Homotopy representations and spheres of representations, Osaka J. Math. 24 (1985), 895-905. MR 87d:57031

[17] Linearity of homotopy representations II, Manuscripta Math. 82 (1994), 277-292. MR 94m:57071

[18] Unstable jO-groups and stably linear homotopy representations for p-groups, Osaka J. Math. 36 (1999), 1-16. MR 99m:57033

[19] R. Oliver, Smooth compact Lie group actions on disks, Math. Z. 149 (1976), 71-96. MR 54:11369

[20] P. Traczyk, On the G-homotopy equivalence of spheres of representations, Math. Z. 161 (1978), 257-261. MR 58:13025

Department of Mathematics, Graduate School of Science, Osaka University, ToyONAKA 560-0043, OSAKA, JAPAN

E-mail address: nagasaki@math.sci.osaka-u.ac.jp 